\title{
Wavelet Spectral Techniques for GPS Errors Reduction
}

\author{
Mohamed Elhabiby, Ahmed El-Ghazouly and Naser El-Sheimy \\ Mobile Multi-Sensor Systems (MMSS) Research Group, \\ Department of Geomatics Engineering, the University of Calgary \\ Calgary, Alberta, CANADA
}

\begin{abstract}
GPS measurements can be modeled as a true range plus other errors such as orbital and clock biases, atmospheric residual, multipath, and observation noise. Modeling is one approach to deal with some of these errors, if their characteristics are known (e.g. troposphere and ionosphere errors). Another way to deal with these errors is filtering in the frequency domain, where all these errors have different frequency spectrum component. Each errors is characterized by a specific frequency band, e.g. the receiver noise can be characterized with high frequency components, multipath errors, which have low to medium frequency bands, while the ionospheric and tropospheric errors are at a lower frequency band.
\end{abstract}

Wavelet spectral techniques can separate GPS signal into sub-bands where different errors can be separated and mitigated. This paper introduces two new wavelet spectral analysis techniques to mitigate DGPS errors in the frequency domain namely, cycle slip and multipath errors. The first approach in this paper, Wavelet de-trending, is introduced to remove the long wavelength carrier phase multipath error in the measurement domain. The presented wavelet-based trend extraction model is applied to GPS static baseline solutions. The second approach in this paper is introduced to detect and remove cycle slip error which can be seen as a singularity in the GPS data. The propagation of singularities between the wavelets levels of decomposition is different from the propagation of noise. This characteristic is used to identify the singularities from noise.

\section{INTRODUCTION}

Wavelets analysis is a powerful developed mathematical tool that has been successfully used in overabundance of applications. Wavelets are extensively used as an alternative to FFT analysis because wavelets elements are waveforms indexed by three parameters (position, scale and frequency). That's the reason of its good localized time-frequency properties, which allow the wavelets to provide an accurate location of the transient component in the signal while retaining information about the fundamental frequency. Therefore, wavelet transform offers advantages over the frequency domain analysis
(Fourier analysis) and the time domain analysis (Kalman filter). Using wavelets, to transform the GPS measurements into frequency domain, helps in localizing both errors location and frequency, this leads to easy error separation in frequency domain.

This paper addresses two GPS errors namely; multipath and cycle slip errors along with their characteristics in the multi-resolution frequency domain. The researsh paper is starting with a brief description and literature review of both GPS errors. After that the background of different wavelet techniques used to mitigate these errors is discussed. Finally, the performance of the proposed techniques will be tested over GPS code and phase measurements.

Under harsh envioroment, i.e. high tropospheric activity or urban areas, GPS carrier phase signal can be lost or blocked casing a cycle slip by integer number of cycles. Cycle slip can be seen as a points of sharp variations in the GPS data, which needs to be detected and removed. Points of sharp variations are mathimaticaly expressed by singularities, which indicate the local regularity of a function. Recently a lot of mathimatical solution are introduced to identify and isolate singularities among of them the Continuous Wavelet Transform (CWT). The capability of Continuous Wavelet Transform in decomposing a signal into frequency bands that are well localized in both time and frequency domains is the reason that CWT performs well in sigularity detection and removal. One of the advantages of CWT is that it gives redundant information between levels of decomposition that should be used to link the singularity between scales. Because of this property, the CWT is capable of defining the local regularity of a signal. CWT is inroduced as a technique that can detect cycle slip by Collin and Warnant in 1995. They analysed the performance of CWT cycle slip detection in code minus carrier and duble difference carrier phase domain. Also, they compared the results from the CWT with kalamn filter technique. They found out that there is no initial condition required to detect cycle slip using wavelets, but signal with a lot of noise will cause successive jumps in the coefficients, which will lead to the detection of different faulty cycle slip (false 
alarm). Since Collin and Warnant paper in 1995, extensive scientific research was made to test the ability of CWT to detect singularities, but without any progress in the area of separaton of false alarm, that created from noise, from true singularity. This research paper introduces a new technique that is used to detect cycle slip location and magnitude in signals contaminated with noise.

The signal received by GPS reciver is a combination of the direct line of sight signal and reflected/diffracted signal or the multipath signal. Multipath is a combination of high, medium and low frequencies errors that contaminate carrier and code measurements. Several post processing techniques are investigated by different research groups (Nce and Sahin, 2000; Satirapod and Rizos 2005; Zheng et al. 2005) in both measurement domain (Kalman filters) and frequency domain (FIR filters, wavelet filters). Previous research done on multipath mitigation using wavelet decomposition techniques is focused on mitigation of high frequency multipath error using the wavelet as a de-noising tool. Ogaja and Satirapod (2007), Sousa and Monico (2004) and Stirapod and Rizos (2005) used wavelet multiresolution at different levels of decomposition using Symlet and Daubechies base function to detect and separate high frequency multipath error. The main target of these researches is to distinguish the base function that can perform best and the thresholding technique that can reduce the high frequency multipath error. Wavelet denoising is a good tool for mitigation/reduction of high frequency errors, i.e. high frequency multipath and noise, but in the case of the low frequency multipath error which is the major effect in the multipath error, the wavelet should be used as a de-trending technique. In this paper a new wavelet de-trending technique is introduced to mitigate the medium to low frequency multipath errors.

\section{BACKGROUND}

\section{CONTINUOUS WAVELETS TRANSFORM (CWT)}

The Continuous Wavelets Transform (CWT) of a signal $q(x)$ is the inner product of the signal with the family of function $\psi(x)$ as described in Equation (1) (Elhabiby, 2007).

$$
Q_{W}\left(m, x_{n}\right)=\frac{1}{\sqrt{m}} \int_{-\infty}^{+\infty} q(x) \bar{\psi}\left(\frac{x-x_{n}}{m}\right) d x
$$

$m$ : the scale that describes the oscillation of the daughter wavelets.

$x_{n}:$ is the shifting for the mother or daughter wavelets.
$\psi$, which is the mother wavelets.
$\bar{\psi}:$ is the complex conjugate of $\psi$.

The computed coefficients $Q_{W}\left(m, x_{n}\right)$ are describing the similarities between shifted and scaled version of the base function and the signal. In the CWT technique, wavelet coefficients are calculated for every possible scale which casuses a lot of work and redundancy. This redundancy is required for some application such as singularity detection, but in the majority of applications the large amount of work done at each level of decomposition is not needed. For ease of computer implementation, the discrete wavelet transform (DWT) technique is implemented and Muli_Resolution Analysis (MRA).

\section{MULTI-RESOLUTION ANALYSIS (MRA)}

Multi-Resolution Analysis (MRA) is introduced for the construction of orthogonal wavelet bases and for the fast decomposition of a signal into independent frequency bands through a nested sequence. MRA builds a pyramidal structure using the two types of filters, lowpass (LP) and high-pass (HP) filters. These filters initially act on the entire signal band and allow the decomposition of the signal into a variety of resolution levels. The data at a lower scale values contains high frequency component and the frequency band is gradually reduces through the high level of decomposition. This structure divides the signal into an approximation $c_{n}^{m}$ and successive details $d_{n}^{m}$ parts. Every one-dimensional signal $\mathrm{f}(\mathrm{t})$ can be represented using wavelet base functions as follows (Keller 2004):

Where:

$$
f(t)=\sum_{m \in Z} c_{n}^{(m)} \Phi_{m, n}(t)+\sum_{m \in Z} \sum_{n \in Z} d_{n}^{(m)} \psi_{m, n}(t)
$$

$$
d_{n}^{(m)}=\left\langle f, \psi_{m, n}\right\rangle \quad c_{n}^{(m)}=\left\langle f, \Phi_{m, n}\right\rangle
$$

$d_{n}^{(m)}:$ is detail

$c_{n}^{m}:$ is approximation.

$\psi_{m, \dot{n}}$ is the wavelet function generated from the original mother wavelet $\psi \in L^{2}(\mathbb{R})$.

$m:$ is the scale or level of decomposition.

$n:$ is the shifting or translation integer.

Where: 
In order to mitigate multipath error in the double differenced domain and cycle slip error in the code minus carrier domain, more advanced multi-resolution techniques is required to capture these errors. In case of cycle slip detection and removal Modulus-Maxima and Lipchitz Exponents are introduced to detect the location of singularities and separate them from noise. And wavelet de-trending technique is introduced to mitigate multipath in the double differenced carrier phase measurements.

\section{MODULUS-MAXIMA AND LIPCHITZ EXPONENTS}

A modulus-maxima of $W f(s)$ is point where $W f(s)$ is a locally maxima, where $W f(s)$ is the wavelets coefficients. The maxima should be strict from either left or right. The variable $S$ represents the scale or the level of decomposition of the wavelets transform. The zooming property of wavelets transform allows time frequency localization on the signal structure. At coarse scales, the overall changes in the signal (i.e. low frequency components) can be detected, while at fine scales, the fine structures (i.e. noise and high frequency components) can be seen. This property is useful for detecting important singularity in noisy signals such as cycle slip in noisy carrier phase measurements. The behavior of the modulus-maxima of the wavelets coefficients for the singularities is different from noise as the amplitude of wavelet modulus maxima for the normal noise decreases strongly when the wavelet scale increases. This does not imply that all local maxima at fine scales correspond to an irregularity. But at least at this point the number of coefficients can be reduced by eliminating the coefficients due to noise and retaining the coefficients that are due to singularity. This can be made by connecting the wavelets local maxima between scales and keep the local maxima that is available in the same location through all scales. Also, even if the wavelet local maxima are connected through scales, the amplitude of local maxima must be increased through scales or it will be removed. This led to dramatically reducing the number of local maxima coefficients and the wavelet coefficients will reflect the singularities and some noise.

The definition of Lipchitz exponent as described in (Mallat and Hwang, 1992) is as follow:

Let $\mathrm{n}$ be a positive integer and $n<\alpha \leq n+1$. A function $f(x)$ is said to be Lipchitz $\alpha$, at $x_{0}$, if and only if there exists two constants $A$ and $h_{0}>0$, and a polynomial of order n, $P_{n}(x)$, such that for $h>h_{0}$

$\left|f\left(x_{0}+h\right)-P_{n}(h)\right| \leq A|h|^{\alpha}$
Usually the local regularity of function is measured with Lipchitz regularity. The decay of wavelets coefficients with scale determines the exponent $\alpha$ at that event or on that interval and can be written as follows (Mallat and Hwang, 1992):

$$
|W f(S)| \leq A S^{\alpha}
$$

Where $A$ is a constant.

In common use of dyadic scale of wavelets transform, equations above becomes:

$\log _{2}|W f(S)| \leq \log _{2} A+\alpha \log _{2}(S)$

Most of the noise in the signal is seen in the first three levels of decomposition and this can deteriorates the value of the wavelets coefficients modulus-maxima at the first level of decomposition. The values of the modules maxima can be retrieved even after the noise deterioration it. This can be done using Equation (6) by estimating the Lipchitz Exponent $\alpha$ of wavelets local maxima between successive scales. Using inverse wavelet transform this value can be used to estimate the singularity.

The technique mentioned above will get rid of most of the singularities due to noise, but some of the wavelet coefficients due to noise can behave like singularities and propagate through scales. These points can be distinguished from true singularities by thresholding the wavelet coefficients based on the signal to noise ration and scale. The threshold is computed from Equation (7) to remove these points (Zhao et al., 2000).

$T_{o}=\frac{\log _{2}(1+2 \sqrt{N})}{J+Z} \cdot K$

Where $N$ is noise power, $J$ is the largest scale selected, $Z$ is a constant, let it be 2 and $K$ is the maximal amplitude of modulus-maxima at the level of decomposition in hand.

\section{WAVELET DE-TRENDING}

The low frequency part of the multipath is superior over high frequency error in the amplitude. Dealing with this fact using the well known de-noising techniques will result in 30-40\% error reduction. This due to the fact that wavelet de-noising techniques are applied in the details coefficients to reduce the high frequency band error. Therefore, to remove the low frequency error, 
thresholding in the approximation part is needed. A new approach based on wavelets de-trending technique is developed by the authors to remove the long wavelength carrier phase multipath error in the measurement domain (Elhabiby et al., 2008).

In order to mitigate low frequency multipath, adaptive wavelet multi-resolution analysis is applied over the GPS double difference observables. As the error is in the low frequency part, wavelets transform approach is used to separate the low frequency multipath error at the high levels of decomposition. The separated wavelet coefficients (approximations or low-level decomposition coefficients) are eliminated by setting the approximation coefficients to zero. This truncation procedure is named "kill approximation thresholding". Finally the details part of the signal is reconstructed to acquire the true double difference carrier phase residuals out of the low frequency multipath.

\section{EXPERIMENT}

In November $9^{\text {th }}, 2007$ an experiment was conducted at university of Calgary to test the ability of the introduced technique to mitigate multipath low frequency part and detect cycle slips. NovAtel's receivers and antennas were used as reference and rover station at the University of Calgary.

Table 1 shows the list of equipment used in the data collection, which started in November $9^{\text {th }}, 2007$ and lasted for five days at the rate of one second data interval the total data collected is 7200 epoch per day. The distance between the two stations is around 100 meters to guarantee the elimination of all the correlated errors, such as ionosphere and troposphere errors, when applying the double difference measurements (Hofmann et al. 2001).

Figure 1 shows the base station at the CCIT building where a clear view with no obstruction in range of 300 meters can be seen. The rover antenna and two receivers are installed on the roof of the engineering building; this configuration is known as the zero baseline. The zerobaseline residuals are used as a reference for comparison.

\begin{tabular}{|c|c|c|c|c|c|c|}
\hline Station & $\begin{array}{c}\text { Zero } \\
\text { Baseline }\end{array}$ & Receiver & Antenna & \multicolumn{2}{|c|}{ Observation } & \begin{tabular}{|c|} 
Duration \\
(Hour)
\end{tabular} \\
\hline \multirow{3}{*}{ Reference } & \multirow{3}{*}{ No } & \multirow{3}{*}{ 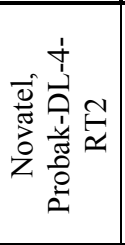 } & \multirow{3}{*}{ 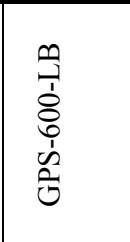 } & $\begin{array}{c}09- \\
\text { Nov }\end{array}$ & Day01 & 2 \\
\hline & & & & $\begin{array}{l}12- \\
\text { Nov }\end{array}$ & Day04 & 2 \\
\hline & & & & $\begin{array}{l}13- \\
\text { Nov }\end{array}$ & Day05 & 2 \\
\hline \multirow{3}{*}{ Rover } & \multirow{3}{*}{ Yes } & \multirow{3}{*}{ 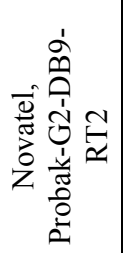 } & \multirow{3}{*}{ 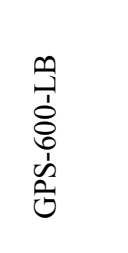 } & $\begin{array}{c}09- \\
\text { Nov }\end{array}$ & Day01 & 2 \\
\hline & & & & $\begin{array}{c}12- \\
\text { Nov }\end{array}$ & Day04 & 2 \\
\hline & & & & $\begin{array}{l}13- \\
\text { Nov }\end{array}$ & Day05 & 2 \\
\hline
\end{tabular}

Table 1. Observation dates and equipments used for reference and rover measurements.

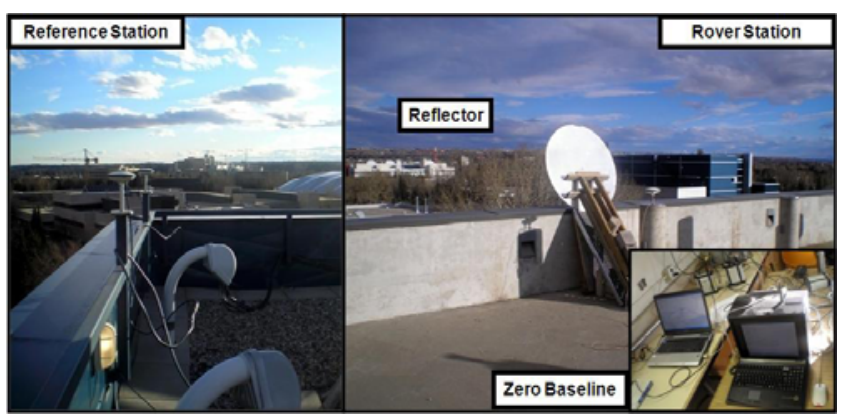

Figure 1. Reference and rover antennas, tow receiver at the rover setup.

\section{DATA WITH SIMULATED CYCLE SLIPS}

To demonstrate the performance of the proposed singularity detection technique, reference and rover L1 pseudorange and carrier phase observations are combined to produce code minus carrier measurements. Only 3000 seconds of measurements are used in this analysis. Different cycle slips are simulated in the measurements at 5 epochs as seen in Table 2.

\begin{tabular}{|c|c|c|c|c|c|c|c|}
\hline Epoch & 0 & 500 & 1000 & 1500 & 2000 & 2500 & 3000 \\
\hline \multirow{2}{*}{ PH1 } & 0 & 0 & 1 & 2 & 3 & 2 & 0 \\
\cline { 2 - 8 } & 0 & 0 & 0.19 & 0.38 & 0.57 & 0.38 & 0 \\
\hline
\end{tabular}

Table 2. Simulated cycle slips 


\section{CYCLE SLIPS DETECTION AND REMOVAL}

The proposed wavelet singularity detection technique is applied on code minus carrier observables. First, CWT is used to decompose the code minus carrier measurements to at scales 2, 4, 8 and 16, which corresponds to level 1,2, 3 and 4. After that, as seen in Figure (2) the modulusmaxima are computed at each of the four scales. The figure is divided into two parts where the upper part shows the original signal and the simulated cycle slips corresponding to Table 2. The computed modulusmaxima over the length of the signal from 1 to 3000 seconds are seen in the lower part of the figure.

It can be seen from Figure (2) magnitude of the modulusmaxima of true singularities at 1000, 1500, 2000 and 2500 second over level of decomposition is higher than the ones that are due to noise. Also, as the scale increases the noise is reduced and signal to noise ratio is increased.
Moreover, faulty singularities due to high noise have large amplitude modulus-maxima at level 4 and 3, which conflict with true singularities. These large amplitude coefficients can be isolated and removed by connecting modulus-maxima between scales as describe above. This will reduce the number of modulus-maxima that not correspond to singularities. But there will be some remaining modulus-maxima that not correspond to singularities and propagate through scales leading to wrong singularity detection (false alarm). Therefore, Equation (7) is used to compute a threshold value based on the scale and the signal to noise ratio. Any wavelet coefficients that have amplitude lower than this value is eliminated and the coefficients that are larger than this threshold are kept in the reconstruction procedure. As a result, the faulty singularities are removed and the true singularities are distinguished in the wavelet coefficients above the noisy and faulty coefficients.
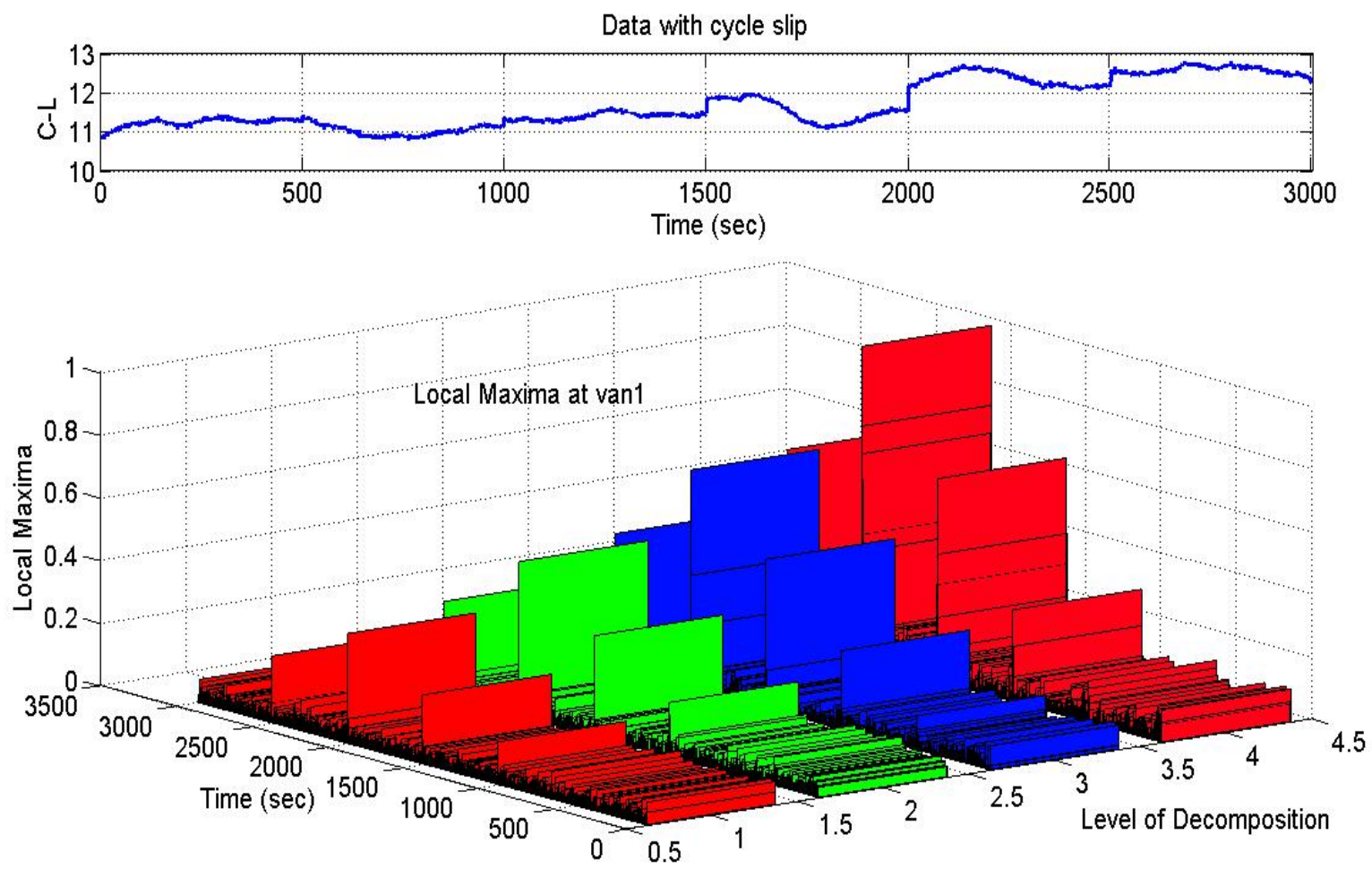

Figure 2. Modulus-maxima at scale 2, 4, 8 and 16 (Level 1, 2, 3 and 4).

Figure (3) shows the results after applying the above procedure to detect and remove singularities. It can be seen that the proposed technique successfully isolates and removes wavelet coefficients corresponding to noise and faulty singularities, while the coefficients for the true singularities are kept untouched. The final step in this procedure is to recover the value of true singularities from the wavelet coefficients. In this step the Lipchitz Exponent is estimated between level 1 to 4 at the epochs $1000,1500,2000$ and 2500 seconds. This is done to recover the singularities coefficients at the first level of decomposition and account for the effect of noise at that level. The estimated Lipchitz Exponents is applied on the coefficients of the last three scales to interpolate the 
modulus-maxima at level 1. Finally, inverse wavelet transform is applied on the interpolated wavelet coefficients at level one to reconstruct the amplitudes of the singularities.

The reconstructed cycle slips are compared with the true simulated ones as shown in Table 3 . It can be seen from the table that the proposed technique can recover up to $98 \%$ of the simulated cycle slip.

\begin{tabular}{|c|c|c|c|c|c|c|c|}
\hline Epoch & 0 & 500 & 1000 & 1500 & 2000 & 2500 & 3000 \\
\hline True & 0 & 0 & 0.19 & 0.38 & 0.57 & 0.38 & 0 \\
\hline Computed & 0 & 0 & 0.23 & 0.385 & 0.58 & 0.34 & 0 \\
\hline$\%$ & 0 & 0 & 83 & 98 & 98 & 90 & 0 \\
\hline
\end{tabular}

Table 3. True and computed cycle slip.
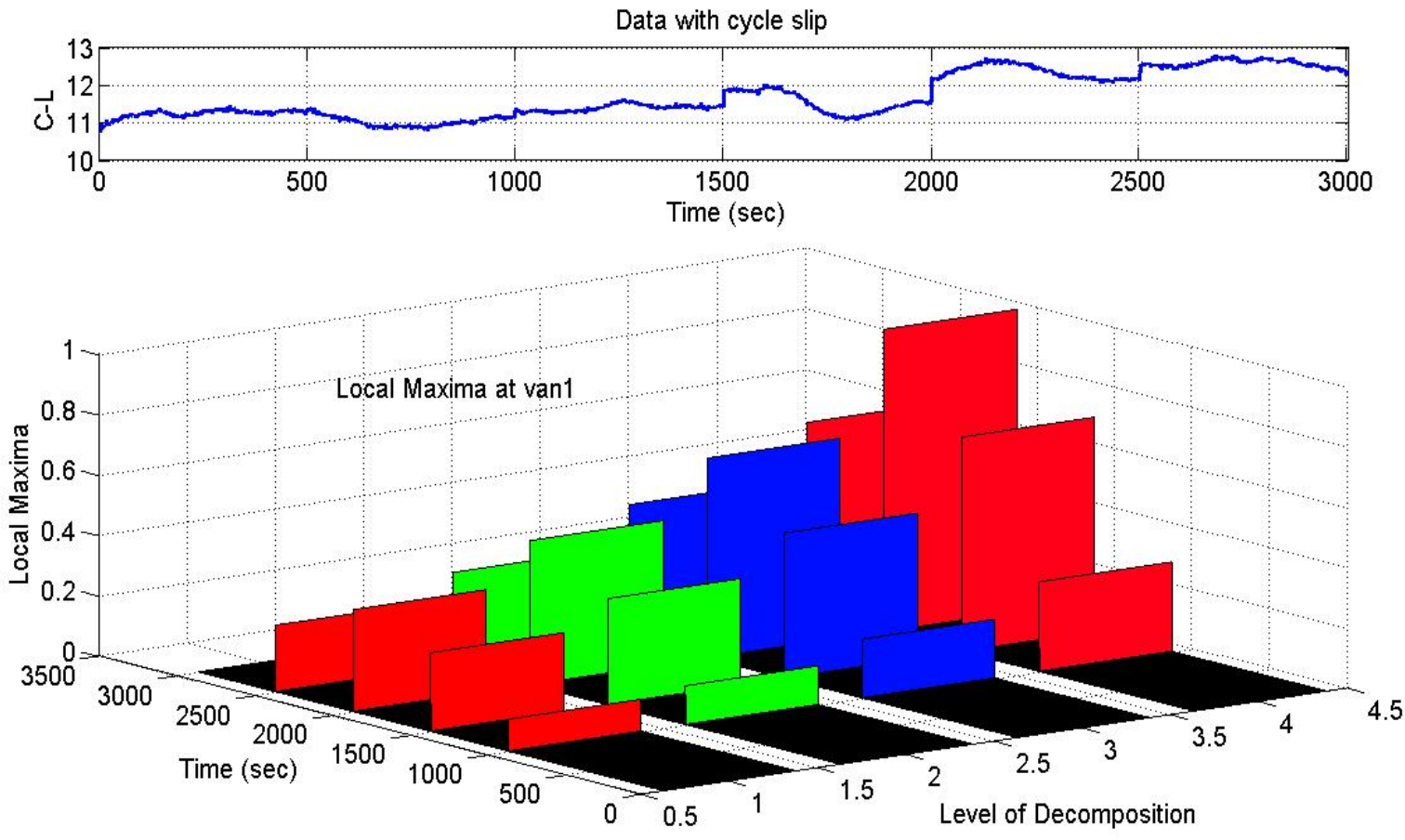

Figure 3. Corrected modulus-maxima at scale 2, 4, 8 and 16 (Level 1, 2, 3 and 4).

\section{MULTIPATH MITIGATION}

In this experiment, there were six satellites-SV02, SV06, SV07, SV22, SV24 and SV31-in view for both the reference and the rover stations. SV06 is chosen to be the reference satellite as it is the highest elevation satellite in this observation window. The results shown here are to the double difference residuals between SV06 with both SV02 and SV05 only as the analysis for all the double difference residuals for the rest of satellites gave identical results. The effect of low frequency multipath can be seen in Figure 4, where the double difference residuals for SV06 as reference and both SV02 and SV05 are plotted. As described before the double differenced measurements are introduced to the proposed wavelet multi-resolution de-trending technique to decompose it into details and approximations.
The Daubechies base function with one vanishing moments is used in the decomposition procedure. The kill approximation thresholding procedure described above is applied to the approximation at the first level of decomposition to mitigate the low frequency multipath error. The reconstructed double difference residuals after mitigation of low frequency multipath error is shown in Figure 5. From this figure a total error reduction up to $78 \%$ is achieved by the wavelet de-trending technique in the double differenced mode. To assess the performance of the wavelet de-trending technique, the corrected double difference measurements is used to compute the rover position in the Earth Centered Earth Fixed (ECEF) frame as shown in Figure 6. 

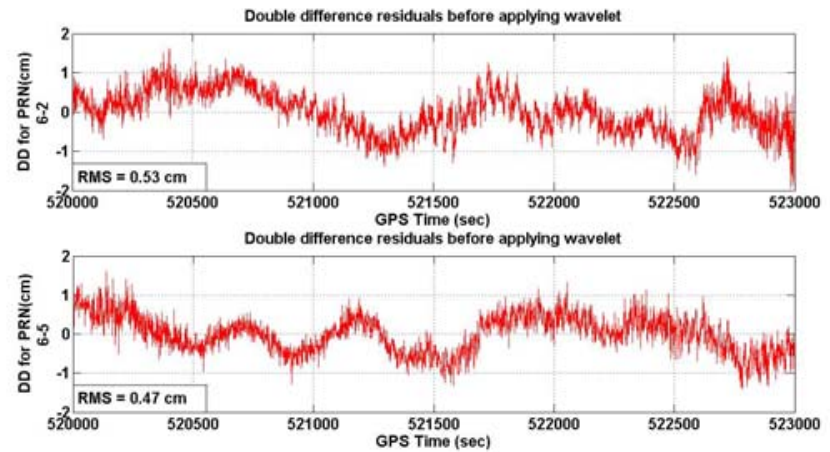

Figure 4. Double difference residuals between satellite SV06 (reference satellite) with satellite SV02, upper part, and satellite SV05, lower part.
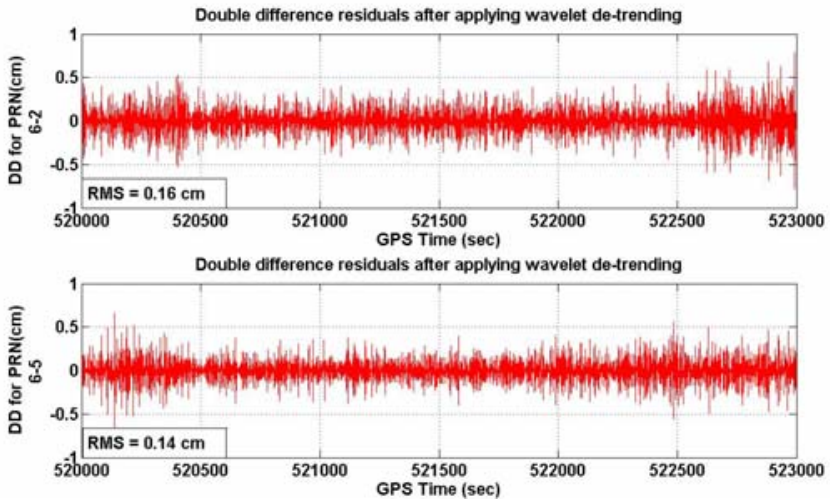

Figure 5. Double difference residuals after applying wavelet technique between satellite SV06 (reference satellite) with satellite SV02, upper part, and satellite SV05, lower part.

The double differenced observation is introduced to least squares estimation technique to estimate the rover position. As can be seen in Figure 4, the double difference observation are contaminated with low frequency multipath error, which will be propagated through the least squares estimation procedure and showed up in the final coordinate domain as a low frequency trend (Figure 6). As can be seen from Figure 6, the error variation in the coordinates domain is in the range of $4 \mathrm{~cm}$ with RMS of 4, 8 and $6 \mathrm{~mm}$ in X, Y and Z directions. Applying wavelet de-trending technique to the coordinates domain led to low frequency error elimination as shown in Figure 7 (upper part). Moreover, the least squares estimation procedure is applied to the corrected double differenced measurements to produce a direct corrected position as shown in the lower part of Figure 7. It can be stated from this figure that the proposed wavelet de-trending technique can successfully used to mitigate the low frequency multipath error in the measurement domain.

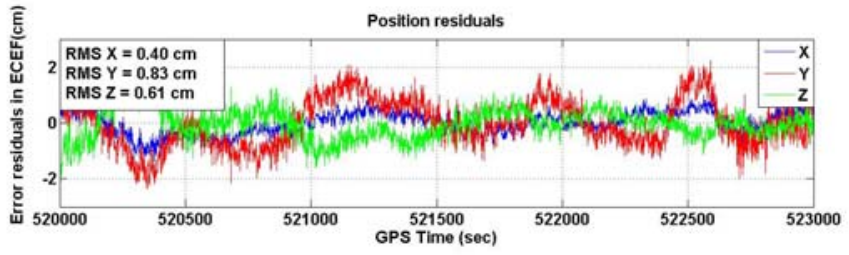

Figure 6. Position residuals in the ECEF frame.

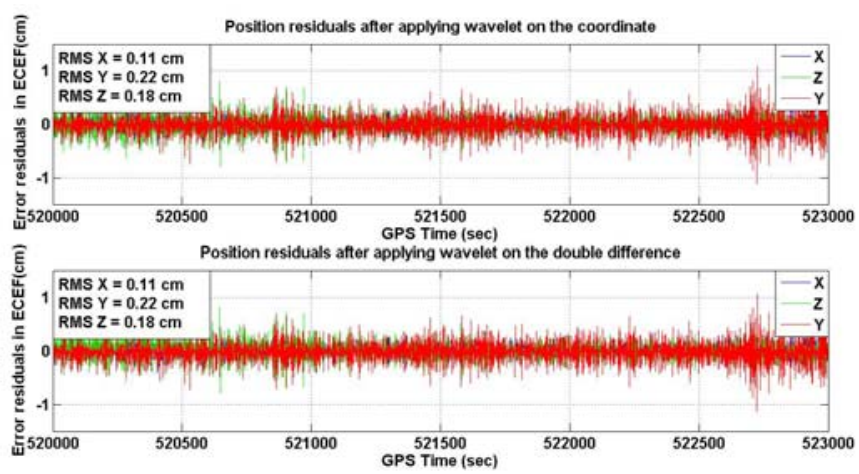

Figure 7. Position residuals in the ECEF frame after applying wavelet technique.

\section{CONCLUSIONS:}

This paper presents two wavelet developments to mitigate GPS errors in the frequency domain. A new wavelets technique combined with Lipchitz Exponents is introduced to detect and remove cycle slip. The proposed technique can efficiently detect the positions of cycle slips and separate it from noise and faulty slips. This technique was successfully tested on real code minus carrier measurements and simulated cycle slips to recover up to $98 \%$ of the amplitude of the cycle slip. More investigation for the performance of the proposed technique to detect and eliminate cycle slips under different signal to noise ratios is needed. The second wavelet multi-resolution technique introduced in this paper is the wavelet detrending technique. The performance of the de-trending technique is investigated over real GPS observations in both the measurements and position domain. The proposed innovative wavelet multi-resolution technique led to a dramatic improvement in the double difference residuals domain and the final coordinate domain to almost $87 \%$. 


\section{REFERENCES}

Chui, C. K. Ed., Wavelets. A tutorial in theory and applications, 1992.

Collin, F. and R. Warnant, Application of the Wavelet Transform for GPS Cycle Slip Correction and Comparison with Kalman Filter, Manuscripta Geodaetica, 20(3), 161-172, 1995.

Daubechies, Ten Lectures on Wavelets, PA SIAM, 1992.

David F. Walnut, An Introduction to wavelet Analysis, Birkhauser Boston, 2002.

Donoho, D.L., Johnstone, I.M. Ideal spatial adaptation by wavelet shrinkage. Biometrika, vol 81, pp. 425-455. 1994.

Elhabiby, M., Wavelet Representation of Geodetic Operators, Ph.D. University of Calgary. Calgary., 2007.

Elhabiby, M., El-Ghazouly, A., El-Sheimy, N. A New Wavelet-Based Multipath Mitigation Technique. ION GNSS 2008, Session C2: Multipath, September 16-19, in Savannah, Georgia. 2008.

Mallat, S. and W. L. Hwang, Singularity detection and processing with wavelets, IEEE Transactions on Information Theory, 38(2), 617-643, 1992.

Zhao, J., Y. Ma, H. Gao and J. Yang, Denoising Method of AE Signal by Using Wavelet Transform, Proc. of 15 th World Conf. on NDT, Rome, 2000.

Grewal, M.S. Angus, L. W., and Andrews, P. Global Positioning Systems, Inertial Navigation, and Integration. WILEY-INTERSCIENCE. USA. 2007.

Hofmann-Wellenhof. B., Lichteneeger, H., and Collins, J. Global Positioning System: Theory and Practice. 5th revised edition. Springer Wien New York. 2001.

Keller, W. Wavelets in geodesy and geodynamics. Walter De Gruyter Inc. 2004.

Leick. A. GPS Satellite Surveying. 3rd ed. John Wiley and Sons, New Jersey. 2004.

Mallat, S.. A wavelet tour of signal processing. Academic Press, New York, USA. 1998
Nce, CD., and Sahin, M. Real-time deformation monitoring with GPS \& Kalman filter. Earth Planets Space 52(10):837-840. 2000.

Ogaja, C., and Satirapod, C. Analysis of high-frequency multipath in $1-\mathrm{Hz}$ GPS kinematic solutions. GPS Solutions 11(4), PP. 269-280. 2007.

Ogden, R. T. Essential wavelets for statistical applications and data analysis. Birkhäuser, Boston, USA. 1997.

Satirapod, C., and Rizos, C. Multipath mitigation by wavelet analysis for GPS base station applications. Survey Review 38(295): 2-10. 2005.

Souza, E.M., and Monico, J.F.G. Wavelet Shrinkage: High frequency multipath reduction from GPS relative positioning, GPS Solutions. 2004.

Zhong, P., Ding, XL., and Chen, W. Filtering GPS timeseries using a Vondrak filter and cross-validation. J Geod 79:363-369. 2005. 